\title{
Wie Ultraschall und Instagram zusammenpassen
}

Browsen Sie gerne durch Instagram? Dann haben Sie bestimmt schon den ein oder anderen radiologischen Account entdeckt. Professor Dirk-André Clevert vom Klinikum der Universität München/Klinik und Poliklinik für Radiologie stellt zum Beispiel mit seinem InstagramAccount ultrasound_campus, gemeinsam mit nationalen und internationalen Kolleginnen und Kollegen, spannende Ultraschallfälle vor. An den neuen Vorsitzenden der AG Ultraschall in der Deutschen Röntgengesellschaft und derzeitigen Vorsitzenden der Sektion Radiologie der Deutschen Gesellschaft für Ultraschall in der Medizin (DEGUM), haben wir aus diesem Grund 3 Fragen rund um Instagram gestellt.

Herr Professor Clevert, die Radiologie ist bildstark, dennoch sind radiologische Accounts auf Instagram noch eher die Ausnahme. Wie kamen Sie auf die Idee, mit Ihrem Account ultrasound_campus online zu gehen?

Gemeinsam mit unseren klinischen Partnern am Klinikum Großhadern sowie mit nationalen und internationalen Kolleginnen und Kollegen bieten wir seit mehr als 16 Jahren regelmäßige interdisziplinäre Ultraschallkurse am Klinikum an. Im Rahmen dieser kontinuierlichen Aus- und Weiterbildung sowie in vielen persönlichen Gesprächen wurde von den Teilnehmerinnen und Teilnehmern vermehrt der Wunsch geäußert, auch nach einem absolvierten Kurs weiterhin trainieren zu können. Im Rahmen der Covid-Pandemie wurde die Anzahl von Präsenzveranstaltungen deutlich

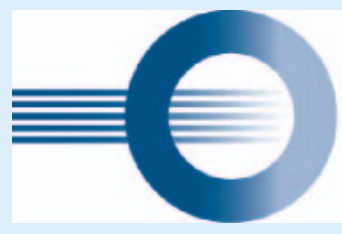

reduziert, aber selbstverständlich war der Bedarf an einer Aus- und Weiterbildung weiterhin vorhanden. Deshalb stellte sich die Frage, wie wir dieses Problem unter Einbezug neuer Medien lösen können. Im Rahmen der zunehmenden Digitalisierung war der logische Schritt, diese Medien, wie etwa die sozialen Netzwerke, vermehrt in die Fortbildung zu integrieren.

Mit mehr als 1400 Followerinnen und Followern konnten Sie bisher eine beachtliche Reichweite aufbauen. Wie haben Sie das geschafft? Hatten Sie Unterstützung?

Der Erfolg verteilt sich natürlich mit nationalen und internationalen Gesellschaften auf viele Schultern, die uns direkt, aber auch indirekt bei diesem Projekt unterstützt haben. Ich möchte hier ein Beispiel heranziehen: Auf Einladung der russischen Gesellschaft für Ultraschall konnten wir mit einem Onlinebeitrag an der 30. Jahrestagung in Moskau teilnehmen. Bereits 2 Tage nach unserem Webinar konnten wir einen deutlichen Anstieg von mehr als 100 neuen Followerinnen und Followern verzeichnen. Weitere Gesellschaften, die uns bei Webinaren ermöglichten, auf unseren Instagram-Account hinzuweisen, waren die Deutsche Röntgengesellschaft
(DRG), die European School of Radiology (ESOR), die Philippine Society of Ultrasound in Clinical Medicine (PSUCMI) und die International Contrast Ultrasound Society (ICUS).

\section{Welche weiteren Pläne verfolgen Sie mit Ihrem Account?}

Der Ultraschall wird weltweit genutzt, jedoch stellt die Ausbildungsproblematik viele Ärztinnen und Ärzte vor Herausforderungen. Wir haben neben unseren lokalen Followerinnen und Followern auch Anfragen aus dem asiatischen Raum, Südamerika und Russland, die diese Möglichkeit der Fortbildung nutzen möchten. Gemeinsam mit der International Contrast Ultrasound Society (ICUS) ist zum Beispiel unser Ziel, die Reichweite zu erhöhen. Diese liegt zum Beispiel in den USA bei 4,7 Prozent. Neben den klassischen organbasierten CaseStudies, die wir in einem ersten Schritt aufund ausgebaut haben, werden wir in einem zweiten Schritt Tutorials zum systematischen Untersuchungsgang ergänzen.

Vielen Dank für das Gespräch und weiterhin viel Erfolg mit ultrasound_campus, Herr Professor Clevert! 